\title{
Microinjection of Antisense Oligonucleotides into Living Mouse Testis Enables LncRNA Function Study
}

\section{Zhaohui Chen}

Nanjing Medical University

\section{Li Ling}

Nanjing Medical University

\section{Xiaolian Shi}

Nanjing Medical University

\section{Wu Li}

Nanjing Medical University

\section{Huicong Zhai}

Nanjing Medical University

\section{Zhenlong Kang}

Nanjing Medical University

\section{Bangjin Zheng}

Nanjing Medical University

Jiaqi Zhu

Nanjing Medical University

\section{Suni Ye}

Nanjing Medical University

Hao Wang

Nanjing Medical University

Lingxiu Tong

Nanjing Medical University

Chaoyang Huang

Zhejiang University School of Medicine

\section{Yang Li}

Nanjing Medical University

Ke Zheng ( $\nabla$ kezheng@njmu.edu.cn )

Nanjing Medical University https://orcid.org/0000-0001-9762-6605 
Keywords: IncRNA (long non-coding RNA), Tsx, ASO (antisense oligonucleotide), knock down, testis injection

Posted Date: July 15th, 2021

DOI: https://doi.org/10.21203/rs.3.rs-707581/v1

License: (c) (1) This work is licensed under a Creative Commons Attribution 4.0 International License. Read Full License

Version of Record: A version of this preprint was published at Cell \& Bioscience on December 1st, 2021. See the published version at https://doi.org/10.1186/s13578-021-00717-y. 


\section{Abstract}

\section{Background}

Long non-coding RNAs (IncRNAs) has been the focus of ongoing research in a diversity of cellular processes. LncRNAs are abundant in mammalian testis, but their biological functions remain poorly known.

\section{Results}

Here, we established an antisense oligonucleotides (ASOs)-based targeting approach that can efficiently knock down IncRNA in living mouse testis. We cloned the full-length transcript of IncRNA TsX (testisspecific X-linked) and defined its testicular localization pattern. Microinjection of ASOs through seminiferous tubules in vivo significantly lowered the Tsx levels in both nucleus and cytoplasm. This effect lasted no less than 10 days, conducive to the generation and maintenance of phenotype. Importantly, ASOs performed better in depleting the nuclear Tsx and sustained longer effect than small interfering RNAs (siRNAs). In addition to the observation of an elevated number of apoptotic germ cells upon ASOs injection, which recapitulates the documented description of Tsx knockout, we also found a specific loss of meiotic spermatocytes despite overall normal process of meiosis.

\section{Conclusions}

Our study detailed the characterization of Tsx and illustrates ASOs as an advantageous tool to functionally interrogate IncRNAs in spermatogenesis.

\section{Background}

Of general curiosity, nearly $98 \%$ of the human genome does not have coding functions [1, 2]. Long noncoding RNAs (IncRNAs) are non-protein coding RNAs longer-than-200 nt which may evolve a regulatory function from 'junk' transcripts[3-5]. With the rapid identification of IncRNAs through deep sequencing comes the urgent demand for the characterization of their biological functions[6-8]. In mammals, transcription of IncRNAs is shown more widespread and dynamic in testis than other organs $[9,10]$. Numerous novel IncRNA genes were discovered in spermatogenic cells, rapidly outpacing the rate of characterizing them[11-14]. Reasons behind this gap include the infrequent emergence of loss-offunction phenotype of IncRNAs as well as the lack of an ideal culturable germline affordable to largescale IncRNA functional screening[15-17].

Employment of various methods such as CRISPR/Cas9-mediated genome editing, RNA interference (RNAi) or antisense oligonucleotides (ASOs) have been reported in IncRNAs loss-of-function studies[1821]. Although the CRISPR/Cas9 system removes transcripts thoroughly compared with the knockdown, it's more laborious, costly and time-consuming. Also, CRISPR/Cas9 is not applicable in some cases. Unlike mRNA, IncRNA function is not prone to be influenced by small deletions or insertions[18, 22]. 
Moreover, genetic manipulation of IncRNAs is likely to perturb the overlapping or adjacent genes. To circumvent such problems, in vivo local knockdown by means of short-hairpin RNAs (shRNAs), small interfering RNAs (siRNAs) or ASOs may be a good alternative. In recent reports, testis transduction of shRNAs in adeno-associated viral (AAV) or lentiviral vectors are demonstrated successful in knockdown of either mRNAs[23, 24] or IncRNAs[14, 25]. siRNA microinjection to achieve transient knockdown effect in young testis has proved a simplified method to examine the meiotic function of protein-coding genes[26]. piRNAs can be targeted by ASOs with the aid of electric transduction in testis[23]. Meanwhile, both siRNAs[27, 28] and ASOs[29-31] have been used for IncRNAs knockdown in vivo other than in mouse testis.

ASOs, differing from siRNAs, are synthesized oligonucleotides with a length of 15-20 nt that can hybridize complementarily with an endogenous RNA to form DNA/RNA heteroduplex and then induce recruitment of the nucleus-enriched RNase $\mathrm{H}$ followed by target RNA cleavage [18, 32]. Pharmacokinetic studies in mice showed that ASOs have long half-lives that can last several weeks[33-35]. Interestingly, nucleus-localized IncRNAs are more easily targeted by ASOs. In knockdown assays on IncRNAs with different subcellular localization, the knockdown efficiency of ASOs for IncRNAs that primarily located in nucleus is higher than that of siRNAs[36]. Moreover, siRNAs-mediated knockdown affects IncRNAs expression solely at post-transcriptional level, while ASOs can also target upon nascent transcripts and block transcription[37]. In some cases, the transcription or splicing process from the transcribed locus rather than the mature IncRNA products confers the IncRNA functionality [7, 38, 39]. Degradation of transcripts carried out by ASOs, rather than siRNAs, can clarify the IncRNA function in its nascent or ontranscription form $[37,40]$. As attractive as the ASO tool is for envisioning its being equivalently applicable in IncRNA knockdown in mammalian testis, a methodological establishment awaits a pioneering attempt.

Considering this technically practical idea a driver of many future investigations to understand IncRNA biological roles in spermatogenesis, we aimed to set up a framework initiated from validating the knockdown effectiveness of testis injection by ASOs through completing a battery of standard phenotypic examinations. To explore ASO application conditions, we first selected a widely expressed, highly nucleus-enriched IncRNA Malat1[41]. For extending to function study, we turned to knock down a testis-specific IncRNA TsX, which represents one rarely documented IncRNA yielding observable phenotype when knocked out in vivo[42, 43]. We defined localization of Tsx and characterized its potential function in sustaining the pool of meiotic germ cells. Comparative assessment of ASOs and siRNAs targeting Tsx highly recommends the former as a better tool for IncRNA function study in testis.

\section{Results}

\section{ASOs-mediated IncRNA knockdown is dose-dependent}

To obtain the optimal ASO injection conditions, we started to inject testis with Malat1 (Metastasis associated lung adenocarcinoma transcript 1) which is highly expressed in GC-2 cells and mouse testis[41]. We first examined the knockdown efficiency of two designed ASOs targeting Malat1 transcripts 
in GC-2 cells by performing fluorescence in situ hybridization (FISH) two days after infection. FISH results showed that the number of Malat1-positive cells was significantly reduced in GC-2 cells transfected with ASO-Malat1-1 (Supplementary Fig. 1). Subsequently, we injected ASO-Malat1-1 into testes of 3-week-old mice and expression of Malat1 was measured by RT-PCR at post-injection 2 and 7 days, respectively. The dilutions of ASOs were directly injected from the ductuli efferentes into seminiferous tubules (Fig. 1A). ASOs with Trypan blue as an indicator filled nearly two third of seminiferous tubules after injection (Fig. 1B). Results of RT-PCR showed that nearly 80\% knockdown efficiency was tested after 2 days of injection at the dosage of $0.08 \mathrm{nmol}$ (Fig. 1C). However, after 7 days, the expression of Malat1 returned to normal levels (Fig. 1C). To maintain the knockdown effect for a longer period of time, we increased the ASO dosage of initial injection. A maximum volume $(4 \mu \mathrm{L})$ of higher amounts of ASOs $(0.2-1 \mathrm{nmol})$ were injected into the testis. More than $1 \mathrm{nmol}$ ASOs would become too viscous to be injected fluently. We collected mouse testis 7 days later for RT-PCR test and determined the range of 0.4-1 nmol ASOs that maintained the knockdown efficacy, with no use for cholesterol modification on ASOs (Fig. 1C).

Next, we turned to test another IncRNA Tsx (testis-specific X-linked). We started with cloning the fulllength Tsx by 5'-end and 3'-end rapid amplification of cDNA ends (RACE) and identified a 818-nt transcript in mouse testis (Supplementary Fig. 2). Three different Tsx-targeting ASOs were injected, among which ASO-TsX-2 was adopted for its highest knockdown efficiency (Fig. 1D). To confirm the best amount for ASO injection, we compared two different amounts $(0.5 \mathrm{nmol}$ and $1 \mathrm{nmol})$ and assessed separately their 10-day knockdown efficiency by FISH. Nearly half tubules of testes injected at 0.5 nmol showed no reduction of $T S X$, while over $80 \%$ of the tubules showed dramatic reduction at $1 \mathrm{nmol}$ (Fig. 1E). No obvious artificial effect was observed between control and Tsx-knockdown testis sections. Therefore, we selected $1 \mathrm{nmol}$ ASO-Tsx-2 for subsequent experiments.

\section{Tsx is distributed in both nucleus and cytoplasm}

Before further evaluating knockdown effect on Tsx, we characterized its expression and localization in postnatal testes. RT-PCR showed that Tsx began to express stably at high levels from 2nd week after birth through the 8-week-old adulthood. (Supplementary Fig. 3A). We then performed co-localization assays by TsX FISH in combination with SOX9 immunofluorescence (IF) on seminiferous tubule sections. The results showed that the punctate signals of IncRNA TsX were partially within the SOX9-positive nucleus of Sertoli cells (Fig. 2A). The rest of Tsx signals spread over from the basal lamina toward lumen, possibly along the cytoplasmic area of Sertoli cells which spaces between germ cells. This speculation was confirmed by co-staining of the Sertoli cytoplasmic marker Vimentin (Fig. 2B). Clearly, Tsx were also shown as individual dots within the SYCP3-marked nucleus of spermatocytes in 2-week-old testis (Supplementary Fig. 3B, indicated by arrows). Moreover, only a few Tsx signals overlapped with the germ cell marker DDX4 (Supplementary Fig. 3C, indicated by arrows), consolidating that the majority of TsX molecules located in the cytoplasmic territory of Sertoli cells but not that of germ cells. Together, Tsx is a nucleocytoplasmic IncRNA in testis.

\section{ASOs, but not siRNAs, persistently inhibit Tsx expression in vivo}


Next, we designed three pieces of siRNAs sequences, and selected one with highest knockdown efficiency for a comparison with ASOs (Fig. 3A). Two days after injection into 3-week-old testes, preparation of subcellular fractions followed by RT-PCR analysis showed better performance of ASOs in nucleus, while both ASOs and siRNAs were able to significantly reduce the cytoplasmic Tsx levels (Fig. 3B). Colocalization of TsX and Vimentin confirmed the presence of considerable amounts of TsX signals in the nucleus of siRNAs instead of ASOs (Fig. 3C, indicated by arrows). For the purpose of achieving sufficient generation of phenotype, we imposed a possibly greatest impact of loss of TsX on germ cell differentiation by prolonging post-injection treatment of testis up to 10 days. As a result, Tsx levels were suppressed around $60 \%$ by ASOs, in contrast with no significance by siRNAs (Fig. 3D). Parallel examination by FISH gave rise to consistent results (Fig. 3E), suggested that ASOs may have more sustainable knockdown effect in vivo than siRNAs.

\section{Tsx inhibition by ASOs raises germ cell defects}

To obtain the stable outcome of Tsx loss-of-function during spermatogenesis, we uniformed a workable time window from executing ASOs injection at postnatal day-21, when meiotic spermatocytes are relatively enriched, to ceasing ASO treatment after 10 days, which represents developmental length of the prophase I of meiosis[44]. TUNEL assays showed a statistically significant rise of apoptotic cells in ASO(Fig. 4A), but not in siRNA-injected testis (Fig. 4B). Our ASO results coincide with the previous description of Tsx knockout mice[43], supporting ASOs a better candidate for Tsx knockdown than siRNAs. To investigate whether Tsx plays a role in meiotic progression, we counted the average numbers of four types of spermatocytes during meiotic prophase I in each seminiferous tubule. Each cell type can be identified based on its correspondence to the specific stages of seminiferous cycle by co-staining of SYCP3, a marker for core composition of synaptonemal complex and PNA, a marker for acrosome (Fig. 4C and D). Compared with control, ASO injection resulted in a statistically significant reduction of zygotene and diplotene spermatocytes (Fig. 4C), albeit no stage exhibited evident morphological abnormality (Fig. 4D). Further, we asked whether loss of Tsx affected meiotic chromosome synapsis and recombination. Various stages of spermatocytes were identified through chromosome spread analysis via co-staining of SYCP1 and SYCP3. No significant defects were observed in the synapsis of homologous chromosomes (Supplementary Fig. 4A). Staining of MLH1 which marks the site of crossover

at late pachytene also showed no difference (Supplementary Fig. 4B and C), neither was the change indicated by the number of SOX9-positive Sertoli cells where most Tsx molecules reside (Supplementary Fig. $5 \mathrm{~A}$ and $\mathrm{B}$ ). In conclusion, Tsx is unnecessary for the overall progression of spermatogenesis, whereas it plays a certain role in the balanced maintenance of germ cells among different types as well as their viability.

\section{Discussion}

It remains mysterious how many IncRNAs act usefully in spermatogenesis. In Drosophila, large-scale knockout screening has yielded dozens of IncRNAs with visual spermatogenic defects[45]. In mice, however, such strategy is neither practicable nor worthy, setting aside that not all types of IncRNAs can be 
specifically or completely knocked out[22, 46]. Few reports have identified functional IncRNAs via creation of individual knockout mouse models[43] or using shRNA-mediated knockdown[14].Unlike shRNA, ASO injection avoids intricate procedures of virus packaging. Common administration ways for ASOs such as intravenous fusion and subcutaneous injection allow for repeated administration[35], which is impractical for seminiferous injection in testis. While our understanding of the in vivo IncRNA physiology stays at its infancy, here we have developed a protocol for microinjecting testis with ASOs to interrogate the function of any individual IncRNAs of interest.

Taking Tsx as an example, ASOs were demonstrated to execute high knockdown efficiency so that testicular cells were largely depleted of its signals and exhibited an induction of apoptosis resembling the described Tsx knockout[43]. Analysis of Tsx levels in subcellular fractions showed that ASOs have better performance than siRNAs in knocking down the nucleus-localized IncRNAs, due possibly to the higher accessibility of ASOs to the nucleus[36], and its unique capability of targeting IncRNAs being transcribed[37]. Compared to siRNAs, moreover, ASOs sustained its effect till post-injection 10 days, a duration long enough to ensure adequate phenotypic analysis. These comparative evaluations support ASOs a better choice for making a knockdown model of IncRNA in living testis. In our study, this TSXdeficiency testis model was used to view the alteration of TsX levels in specific cell types and in different subcellular compartments by FISH and IF double staining. Tsx was previously reported to be distributed at the periphery of the tubule where Sertoli cells usually locate by In situ hybridization (ISH)[42]. We found that $T s x$ is a nucleocytoplasmic IncRNA mainly expressed in Sertoli cells and minorly in germ cells. This model was also used to investigate the meiotic defect by employing standard immunofluorescence analyses of either seminiferous sections or chromosome spreads. Our data revealed the involvement of Tsx in maintaining a balanced pool of spermatocytes. In sum, our ASO-based IncRNA-targeting method, in junction with various follow-up experiments, will facilitate a broad range of investigations to understand the IncRNAs' roles in mammalian male reproduction.

In recent years, RNA therapeutics based on siRNAs, ASOs and CRISPRi (CRISPR interference) that target transcripts of the genes have emerged. CRISPRi is developed from CRISPR system that has been successfully used in preclinical animal tests and ex vivo modification of iPSCs for transplantation[4749]. Compared to the infancy of clinical application of CRISPRi, drugs based on siRNAs and ASOs have emerged and been reported to be used in phase III trials[50-52]. Specifically, unlike siRNAs which need a delivery agent, ASOs can be taken up by cells without any delivery agents and has lower biological toxicity. These merits make ASOs more applicable in clinical trials[53-55]. Besides, ASOs can act in the nucleus and intervene in transcription, which makes ASOs widely used in pre-clinical studies of singlegene disorders[56]. The method we established for ASO-mediated testis knockdown in vivo also offers possibility for pre-clinical application of ASOs in humans with regards to male contraception or improvement of reproductive health [57-59].

\section{Conclusion}


Here we have established an ASO microinjection-initiated procedure that is capable of creating a testisspecific knockdown model for IncRNA. Using this model, along with other experiments, we show that: 1) ASOs efficiently knocked down both the IncRNAs Malat1 and TsX, 2) Tsx is a nucleocytoplasmic IncRNA in testicular cells; 3 ) Tsx can be efficiently and persistently acted upon by ASOs rather than siRNAs; 4) TsX deficiency led to a disturbance to the maintaining of meiotic germ cell pool.

\section{Materials And Methods}

\section{Mice, ASOs, siRNAs and Antibodies}

C57BL/ 6 mice were obtained from the Model Animal Research Center of Nanjing University. All mice were housed with $12 / 12 \mathrm{~h}$ light/dark cycles, at $22^{\circ} \mathrm{C}$ and allowed free access to water and food.

ASOs and siRNAs were synthesized by Ribobio (Guangzhou, China). Specific sequences are provided in supplementary information Table S1. ASOs used in Figure 1C were diluted with nuclease-free water to different concentrations within 20-500 $\mu \mathrm{M}$, and injected into the testis with the maximum injection volume $(4 \mu \mathrm{L})$. siRNAs we used in this article were chemically modified with 2'-OM (2-methoxyethyl) and cholesterol to improve the stability and biodistribution[53].

Primary antibodies used in this article are listed as follow: rabbit anti-Vimentin (ab92547, Abcam, USA), rabbit anti-SOX9 (AB5535, Merck Millipore, German), rabbit anti-DDX4 (ab13840, Abcam, USA) and rabbit anti-SYCP3 (ab15093, Abcam, USA), rabbit anti-SYCP1 (ab15090, Abcam, USA), rabbit anti-MLH1 (550838, BD, USA).

\section{In vivo knockdown through microinjection}

Microinjection of ASOs and siRNAs were operated as previously described[60]. Mice of 3-week-old were anesthetized by tri-bromoethanol and one testis was exteriorized through incisions on abdomen. A mixture of $3 \mu \mathrm{L}$ of ASO dilutions or siRNA dilutions and $1 \mu \mathrm{L}$ tracer Trypan blue was injected into seminiferous tubule through the microinjection apparatus (FemtoJet 4i, Eppendorf) under a stereoscopic microscope. The testis was placed back to the abdominal cavity after injection. After both sides of testes were finished with injection, the incisions were closed with sutures. Each injected mouse was kept warm by putting hot water bag in cage until they wake up.

\section{RNA extraction and RT-PCR}

Testes were collected 48 hours or 10 days after injection to measure knockdown efficiency. Total RNA was extracted using TRIzol reagent (Thermo Fisher Scientific, USA) following the manufacturer's instructions. $1 \mu \mathrm{g}$ RNA was reversed transcribed into cDNA with PrimeScript RT Master Mix (RR036A, TaKaRa, Tokyo, Japan) according to the manufacturer's instructions. Real time PCR was performed using TB Green Premix Ex Taq II(RR820A, TaKaRa, Tokyo, Japan) on Applied Biosystems StepOnePlus RealTime PCR System. Relative expression of target RNA was determined after normalization to 36B4 gene. The sequences of all primers used in this experiment are provided in additional file 2 Table S1. 


\section{Rapid amplification of CDNA ends (RACE) analysis}

Total RNA was extracted from C57BL/ 6 mouse testis. 5'RACE and 3'RACE were performed using the SMARTer ${ }^{\circledR}$ RACE 5'/3' Kit (Clontech, Mountain View, CA) according to the manufacturer's instructions. The following gene-specific primers (GSP) are used for PCR:

5'-TGGCAAGCAACAAACACCCTAGTTGGC-3' (5'RACE GSP);

5'-CTTGGGTATCAGCTCCACCAACAAGGT-3' (3'RACE GSP).

\section{Fluorescence in situ hybridization (FISH) and immunofluorescence}

Testes were fixed in $4 \%$ PFA overnight at $4{ }^{\circ} \mathrm{C}$, dehydrated by graded ethanol $(70 \%, 95 \%, 100 \%)$ and embedded in paraffin. Embedded testes were sectioned into $5 \mu \mathrm{m}$. FISH was performed with RNAscope@ multiplex fluorescent reagent kit (Advanced Cell Diagnostics, USA) as manufacturer's instructions[61]. Target probes were obtained from RNAscope ${ }^{@}$. After the last procedure of FISH, sections were treated in 1×PBS for $5 \mathrm{~min}$ and then blocked in blocker (10\% FBS, 1\% BSA, 1\% Triton X-100, 0.05\% Tween-20) for 1 $\mathrm{hr}$ at room temperature (RT). Testis sections were incubated with the following antibodies at $4{ }^{\circ} \mathrm{C}$ overnight: rabbit anti-Vimentin (1:250), rabbit anti-SOX9 (1:400), rabbit anti-DDX4 (1:200) and rabbit antiSYCP3 (1:100). Texas red or FITC-conjugated secondary antibodies (Vector Laboratories, USA) were incubated at $37^{\circ} \mathrm{C}$ for $1 \mathrm{hr}$ and sections were washed 3 times in PBST ( $1 \times$ PBS containing $0.2 \%$ Tween20). Samples were mounted in microslide shield with DAPI. Fluorescent signals were detected on a confocal microscope (LSM800, Carl Zeiss, German).

\section{Isolation of nuclear and cytoplasmic fractions}

Subcellular extracts were prepared as described[60]. 30-40 mg testis tissue was homogenized in $400 \mu \mathrm{L}$ Cytoplasmic Extraction Buffer (CT Buffer, $250 \mathrm{mM}$ sucrose, $10 \mathrm{mM}$ Tris- $\mathrm{HCl}$ (pH 8.0), $10 \mathrm{mM} \mathrm{MgCl2,} 1$ mM EGTA, $1 \times$ protease inhibitor cocktail III, $0.4 \mu \mathrm{L}$ RNasin (N251B, Promega, USA)) with 100 strokes. The lysis was centrifuged at $300 \times \mathrm{g}$ for $5 \mathrm{~min}$, and the supernatant was collected as cytoplasmic fraction. Add $1 \mathrm{~mL}$ TRIzol reagent per $200 \mu \mathrm{L}$ supernatant and stored at $-80^{\circ} \mathrm{C}$. The pellet seen as nuclei fraction was washed three times in $500 \mu \mathrm{L}$ CT Buffer and centrifuged at $300 \times \mathrm{g}$ for $5 \mathrm{~min}$. Add $1 \mathrm{~mL}$ TRIzol and homogenize the pellet by $1 \mathrm{~mL}$ syringe with $0.4 \mathrm{~mm}$ needle. RNA was extracted as above.

\section{TUNEL assays, chromosome spread and immunofluorescence}

TUNEL was performed on the testis sections with TUNEL BrightGreen Apoptosis Detection Kit (A112-01, Vazyme, China) according to the manufacturer's instructions. Chromosome spread of prophase I spermatocytes were performed as previous described[62]. The following primary antibodies were used in morphology analysis of chromosome: rabbit anti-SYCP1 (1:100), rabbit anti-SYCP3 (1:100), rabbit antiMLH1 (1:100). Slides were washed in 1×PBS for 5 min, and then blocked with $10 \%$ goat serum in PBST ( $1 \times$ PBS containing $0.1 \%$ Tween 20 ) for $1 \mathrm{hr}$ at RT. After incubated with primary antibodies overnight at 4 ${ }^{\circ} \mathrm{C}$, slides were treated with PBST for 3 times. Secondary antibodies were incubated at $37^{\circ} \mathrm{C}$ for $1 \mathrm{hr}$. 
Wash slides in PBST for 3 times. All the slides were mounted in microslide shield with DAPI. Immunofluorescence for all samples was examined under laser scanning confocal microscope (LSM800, Carl Zeiss, German).

\section{Statistical analysis}

All values were presented as mean \pm S.D. Statistical analysis was performed with Student's $t$ test $\left({ }^{\star} p<0.05 ;{ }^{* \star} p<0.01 ;{ }^{* \star *} p<0.001\right)$ using Prism 7.0 (GraphPad Software, La Jolla, CA, USA). NS means not significant.

\section{Declarations}

\section{Ethics approval and consent to participate}

All experiments involving mice were conducted according to the guidelines of the Institutional Animal Care and Use Committee of Nanjing Medical University.

\section{Consent for publication}

Not applicable.

\section{Availability of data and materials}

All data generated or analysed during this study are included in this published article and its supplementary information files.

\section{Competing interests}

The authors declare that they have no competing interests.

\section{Funding}

This work was supported by National Natural Science Foundation of China $(31771653,31970791$ and 82001613); Postdoctoral research foundation of china (2020M671540).

\section{Authors' contributions}

K.Z., Y.L. and C.-Y.H conceived and supervised the project. Z.-H.C., L.L., X.-L. S.,, L.Y. and K.Z. designed and performed most experiments. Z.-L. K., H.-C. Z., W.L., B.-J. Z., J.-Q. Z., S.-N. Y., H.W. and X.-J. T. performed some experiments or provided technical assistance. K.Z. and Z.-H. C. wrote the manuscript with major inputs from L.L., X.-L. S., and L.Y.. All authors approved the final manuscript.

\section{Acknowledgements}


We thank Yanfeng Wang, Mengrou Liu, Fangfang Li (Nanjing Medical University, China) for conferring experimental guidance or help.

\section{References}

1. Djebali S, Davis CA, Merkel A, Dobin A, Lassmann T, Mortazavi A, et al. Landscape of transcription in human cells. Nature. 2012;489(7414):101-8.

2. Pennisi E. Genomics. ENCODE project writes eulogy for junk DNA. Science. 2012;337(6099):1159, 61.

3. Palazzo AF, Koonin EV. Functional Long Non-coding RNAs Evolve from Junk Transcripts. Cell. 2020;183(5):1151-61.

4. Ponting CP, Oliver PL, Reik W. Evolution and functions of long noncoding RNAs. Cell. 2009;136(4):629-41.

5. Yao RW, Wang Y, Chen LL. Cellular functions of long noncoding RNAs. Nat Cell Biol. 2019;21(5):54251.

6. Iyer MK, Niknafs YS, Malik R, Singhal U, Sahu A, Hosono Y, et al. The landscape of long noncoding RNAs in the human transcriptome. Nat Genet. 2015;47(3):199-208.

7. Kopp F, Mendell JT. Functional Classification and Experimental Dissection of Long Noncoding RNAs. Cell. 2018;172(3):393-407.

8. Qian X, Zhao J, Yeung PY, Zhang QC, Kwok CK. Revealing IncRNA Structures and Interactions by Sequencing-Based Approaches. Trends Biochem Sci. 2019;44(1):33-52.

9. Soumillon M, Necsulea A, Weier M, Brawand D, Zhang X, Gu H, et al. Cellular source and mechanisms of high transcriptome complexity in the mammalian testis. Cell Rep. 2013;3(6):2179-90.

10. Watanabe T, Cheng EC, Zhong M, Lin H. Retrotransposons and pseudogenes regulate mRNAs and IncRNAs via the piRNA pathway in the germline. Genome Res. 2015;25(3):368-80.

11. Trovero MF, Rodriguez-Casuriaga R, Romeo C, Santinaque FF, Francois M, Folle GA, et al. Revealing stage-specific expression patterns of long noncoding RNAs along mouse spermatogenesis. RNA Biol. 2020;17(3):350-65.

12. Bao J, Wu J, Schuster AS, Hennig GW, Yan W. Expression profiling reveals developmentally regulated IncRNA repertoire in the mouse male germline. Biol Reprod. 2013;89(5):107.

13. Hong SH, Kwon JT, Kim J, Jeong J, Kim J, Lee S, et al. Profiling of testis-specific long noncoding RNAs in mice. BMC Genom. 2018;19(1):539.

14. Li K, Xu J, Luo Y, Zou D, Han R, Zhong S, et al. Panoramic transcriptome analysis and functional screening of long noncoding RNAs in mouse spermatogenesis. Genome Res. 2021;31(1):13-26.

15. George MR, Duan Q, Nagle A, Kathiriya IS, Huang Y, Rao K, et al. Minimal in vivo requirements for developmentally regulated cardiac long intergenic non-coding RNAs. Development. 2019;146(23).

16. Goudarzi M, Berg K, Pieper LM, Schier AF. Individual long non-coding RNAs have no overt functions in zebrafish embryogenesis, viability and fertility. Elife. 2019;8. 
17. Richer $G$, Baert $Y$, Goossens E. In-vitro spermatogenesis through testis modelling: Toward the generation of testicular organoids. Andrology. 2020;8(4):879-91.

18. Liu SJ, Lim DA. Modulating the expression of long non-coding RNAs for functional studies. EMBO Rep. 2018;19(12).

19. Stojic L, Lun ATL, Mangei J, Mascalchi P, Quarantotti V, Barr AR, et al. Specificity of RNAi, LNA and CRISPRi as loss-of-function methods in transcriptional analysis. Nucleic Acids Res. 2018;46(12):5950-66.

20. Lucere KM, O'Malley MMR, Diermeier SD. Functional Screening Techniques to Identify Long NonCoding RNAs as Therapeutic Targets in Cancer. Cancers (Basel). 2020;12(12).

21. Stojic L, Lun ATL, Mascalchi P, Ernst C, Redmond AM, Mangei J, et al. A high-content RNAi screen reveals multiple roles for long noncoding RNAs in cell division. Nat Commun. 2020;11(1):1851.

22. Li L, Chang HY. Physiological roles of long noncoding RNAs: insight from knockout mice. Trends Cell Biol. 2014;24(10):594-602.

23. Gou LT, Dai P, Yang JH, Xue Y, Hu YP, Zhou Y, et al. Pachytene piRNAs instruct massive mRNA elimination during late spermiogenesis. Cell Res. 2014;24(6):680-700.

24. Dai P, Wang X, Gou LT, Li ZT, Wen Z, Chen ZG, et al. A Translation-Activating Function of MIWI/piRNA during Mouse Spermiogenesis. Cell. 2019;179(7):1566-81 e16.

25. Lei Q, Pan Q, Li N, Zhou Z, Zhang J, He X, et al. H19 regulates the proliferation of bovine male germline stem cells via IGF-1 signaling pathway. J Cell Physiol. 2018;234(1):915-26.

26. Dai J, Voloshin O, Potapova S, Camerini-Otero RD. Meiotic Knockdown and Complementation Reveals Essential Role of RAD51 in Mouse Spermatogenesis. Cell Rep. 2017;18(6):1383-94.

27. Yu Q, Qiu Y, Wang X, Tang J, Liu Y, Mei L, et al. Efficient siRNA transfer to knockdown a placenta specific IncRNA using RGD-modified nano-liposome: A new preeclampsia-like mouse model. Int $\mathrm{J}$ Pharm. 2018;546(1-2):115-24.

28. Sun T, Cheng YT, Yan LX, Krittanawong C, Qian W, Zhang HJ. LncRNA MALAT1 knockdown alleviates myocardial apoptosis in rats with myocardial ischemia-reperfusion through activating PI3K/AKT signaling pathway. Eur Rev Med Pharmacol Sci. 2019;23(23):10523-31.

29. Crooke ST, Wang S, Vickers TA, Shen W, Liang XH. Cellular uptake and trafficking of antisense oligonucleotides. Nat Biotechnol. 2017;35(3):230-7.

30. Wheeler TM, Leger AJ, Pandey SK, MacLeod AR, Nakamori M, Cheng SH, et al. Targeting nuclear RNA for in vivo correction of myotonic dystrophy. Nature. 2012;488(7409):111-5.

31. Xiu B, Chi Y, Liu L, Chi W, Zhang Q, Chen J, et al. LINC02273 drives breast cancer metastasis by epigenetically increasing AGR2 transcription. Mol Cancer. 2019;18(1):187.

32. Wu H, Lima WF, Zhang H, Fan A, Sun H, Crooke ST. Determination of the role of the human RNase H1 in the pharmacology of DNA-like antisense drugs. J Biol Chem. 2004;279(17):17181-9.

33. Yu RZ, Kim TW, Hong A, Watanabe TA, Gaus HJ, Geary RS. Cross-species pharmacokinetic comparison from mouse to man of a second-generation antisense oligonucleotide, ISIS 301012, 
targeting human apolipoprotein B-100. Drug Metab Dispos. 2007;35(3):460-8.

34. Rigo F, Chun SJ, Norris DA, Hung G, Lee S, Matson J, et al. Pharmacology of a central nervous system delivered 2'-0-methoxyethyl-modified survival of motor neuron splicing oligonucleotide in mice and nonhuman primates. J Pharmacol Exp Ther. 2014;350(1):46-55.

35. Geary RS, Norris D, Yu R, Bennett CF. Pharmacokinetics, biodistribution and cell uptake of antisense oligonucleotides. Adv Drug Deliv Rev. 2015;87:46-51.

36. Lennox KA, Behlke MA. Cellular localization of long non-coding RNAs affects silencing by RNAi more than by antisense oligonucleotides. Nucleic Acids Res. 2016;44(2):863-77.

37. Lee JS, Mendell JT. Antisense-Mediated Transcript Knockdown Triggers Premature Transcription Termination. Mol Cell. 2020;77(5):1044-54 e3.

38. Latos PA, Pauler FM, Koerner MV, Senergin HB, Hudson QJ, Stocsits RR, et al. Airn transcriptional overlap, but not its IncRNA products, induces imprinted Igf2r silencing. Science. 2012;338(6113):1469-72.

39. Engreitz JM, Haines JE, Perez EM, Munson G, Chen J, Kane M, et al. Local regulation of gene expression by IncRNA promoters, transcription and splicing. Nature. 2016;539(7629):452-5.

40. Luo S, Lu JY, Liu L, Yin Y, Chen C, Han X, et al. Divergent IncRNAs Regulate Gene Expression and Lineage Differentiation in Pluripotent Cells. Cell Stem Cell. 2016;18(5):637-52.

41. Zhang B, Arun G, Mao YS, Lazar Z, Hung G, Bhattacharjee G, et al. The IncRNA Malat1 is dispensable for mouse development but its transcription plays a cis-regulatory role in the adult. Cell Rep. 2012;2(1):111-23.

42. Cunningham DB, Segretain D, Arnaud D, Rogner UC, Avner P. The mouse Tsx gene is expressed in Sertoli cells of the adult testis and transiently in premeiotic germ cells during puberty. Dev Biol. 1998;204(2):345-60.

43. Anguera MC, Ma W, Clift D, Namekawa S, Kelleher RJ 3rd, Lee JT. Tsx produces a long noncoding RNA and has general functions in the germline, stem cells, and brain. PLoS Genet. 2011;7(9):e1002248.

44. Goetz P, Chandley AC, Speed RM. Morphological and temporal sequence of meiotic prophase development at puberty in the male mouse. J Cell Sci. 1984;65:249-63.

45. Wen K, Yang L, Xiong T, Di C, Ma D, Wu M, et al. Critical roles of long noncoding RNAs in Drosophila spermatogenesis. Genome Res. 2016;26(9):1233-44.

46. Goyal A, Myacheva K, Gross M, Klingenberg M, Duran Arque B, Diederichs S. Challenges of CRISPR/Cas9 applications for long non-coding RNA genes. Nucleic Acids Res. 2017;45(3):e12.

47. Liu SJ, Horlbeck MA, Cho SW, Birk HS, Malatesta M, He D, et al. CRISPRi-based genome-scale identification of functional long noncoding RNA loci in human cells. Science. 2017;355(6320).

48. Peddle CF, Fry LE, McClements ME, MacLaren RE. CRISPR Interference-Potential Application in Retinal Disease. Int J Mol Sci. 2020;21(7). 
49. Kampmann M. CRISPR-based functional genomics for neurological disease. Nat Rev Neurol. 2020;16(9):465-80.

50. Adams D, Gonzalez-Duarte A, O'Riordan WD, Yang CC, Ueda M, Kristen AV, et al. Patisiran, an RNAi Therapeutic, for Hereditary Transthyretin Amyloidosis. N Engl J Med. 2018;379(1):11-21.

51. Rinaldi C, Wood MJA. Antisense oligonucleotides: the next frontier for treatment of neurological disorders. Nat Rev Neurol. 2018;14(1):9-21.

52. Dorsett $Y$, Tuschl T. siRNAs: applications in functional genomics and potential as therapeutics. Nat Rev Drug Discov. 2004;3(4):318-29.

53. Dowdy SF. Overcoming cellular barriers for RNA therapeutics. Nat Biotechnol. 2017;35(3):222-9.

54. Ingusci S, Verlengia G, Soukupova M, Zucchini S, Simonato M. Gene Therapy Tools for Brain Diseases. Front Pharmacol. 2019;10:724.

55. Wild EJ, Tabrizi SJ. Therapies targeting DNA and RNA in Huntington's disease. Lancet Neurol. 2017;16(10):837-47.

56. Beaudet AL, Meng L. Gene-targeting pharmaceuticals for single-gene disorders. Hum Mol Genet. 2016;25(R1):R18-26.

57. Thirumalai A, Page ST. Recent Developments in Male Contraception. Drugs. 2019;79(1):11-20.

58. Rolland AD, Evrard B, Darde TA, Le Beguec C, Le Bras Y, Bensalah K, et al. RNA profiling of human testicular cells identifies syntenic IncRNAs associated with spermatogenesis. Hum Reprod. 2019;34(7):1278-90.

59. Joshi M, Rajender S. Long non-coding RNAs (IncRNAs) in spermatogenesis and male infertility. Reprod Biol Endocrinol. 2020;18(1):103.

60. Fu K, Tian S, Tan H, Wang C, Wang H, Wang M, et al. Biological and RNA regulatory function of MOV10 in mammalian germ cells. BMC Biol. 2019;17(1):39.

61. Wang F, Flanagan J, Su N, Wang LC, Bui S, Nielson A, et al. RNAscope: a novel in situ RNA analysis platform for formalin-fixed, paraffin-embedded tissues. J Mol Diagn. 2012;14(1):22-9.

62. Luo M, Yang F, Leu NA, Landaiche J, Handel MA, Benavente R, et al. MEIOB exhibits single-stranded DNA-binding and exonuclease activities and is essential for meiotic recombination. Nat Commun. 2013;4:2788.

\section{Figures}



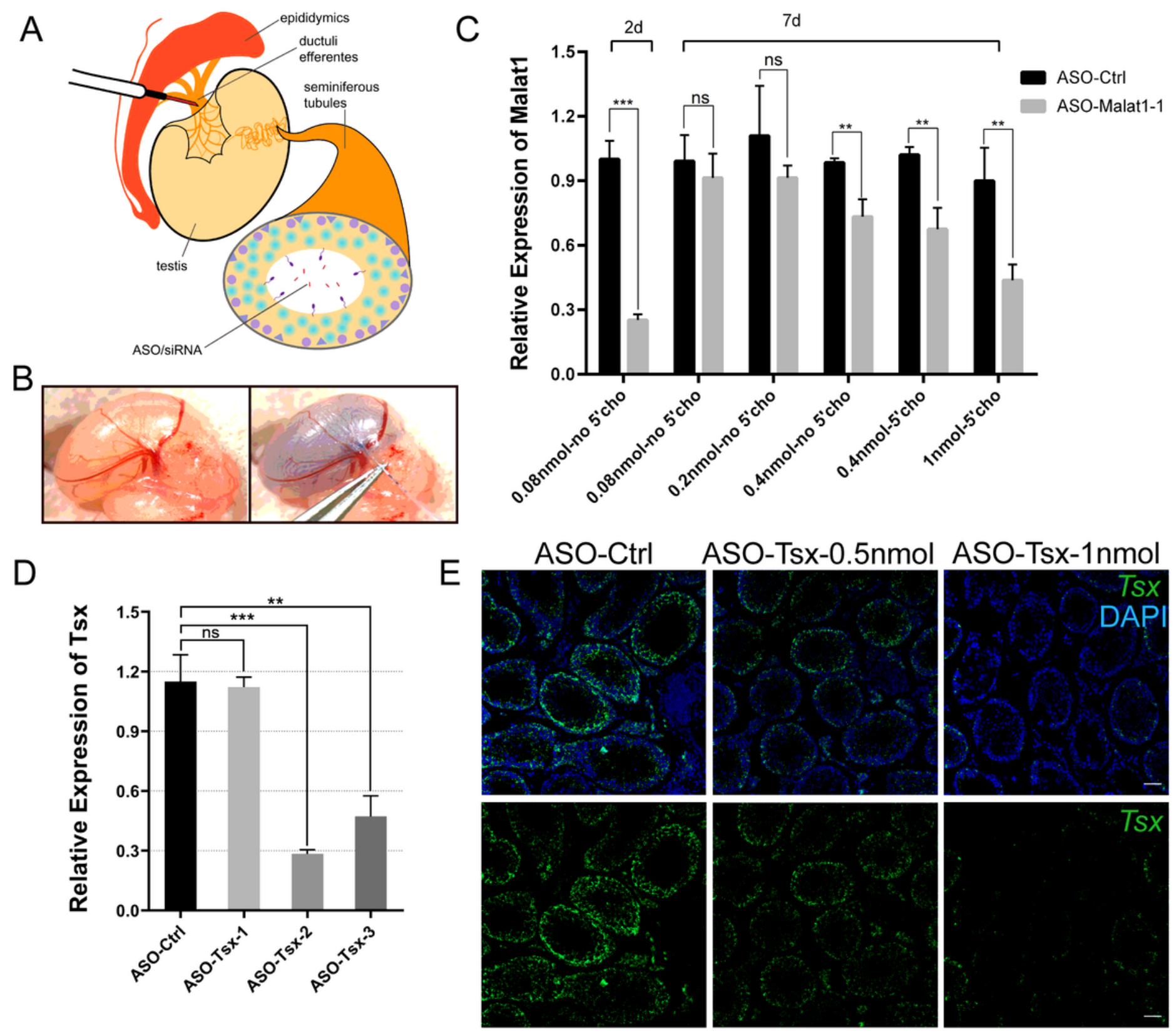

Figure 1

Knockdown efficiency of ASOs in testis. (A) Schematic diagram of microinjection process through seminiferous tubules. (B) Photos of testis from 3-week-old mouse taken before (left) and after (right) injection. Filling of seminiferous tubules was monitored with the help of Trypan blue. (C) Relative expression of Malat1 detected by RT-PCR after injecting different doses of ASO-Malat1-1 into mouse testis. Testes were collected 2 or 7 days after injection. Values are expressed as mean \pm S.D. Statistical significance was determined using t tests. ${ }^{*} p<0.01$ and ${ }^{* \star *} p<0.001$. $n=3$. (D) Relative expression of Tsx in testis measured by RT-PCR 2 days after injection of three different ASOs. Values are expressed as mean $\pm S$.D. Statistical significance was determined using $t$ tests. ${ }^{\star *} p<0.01$ and ${ }^{* \star \star} p<0.001$. $n=3$. (E) Tsx RNA (green) detected by FISH in testes injected by different doses of ASO-Tsx-2. Scale bar, $50 \mu \mathrm{m}$. 


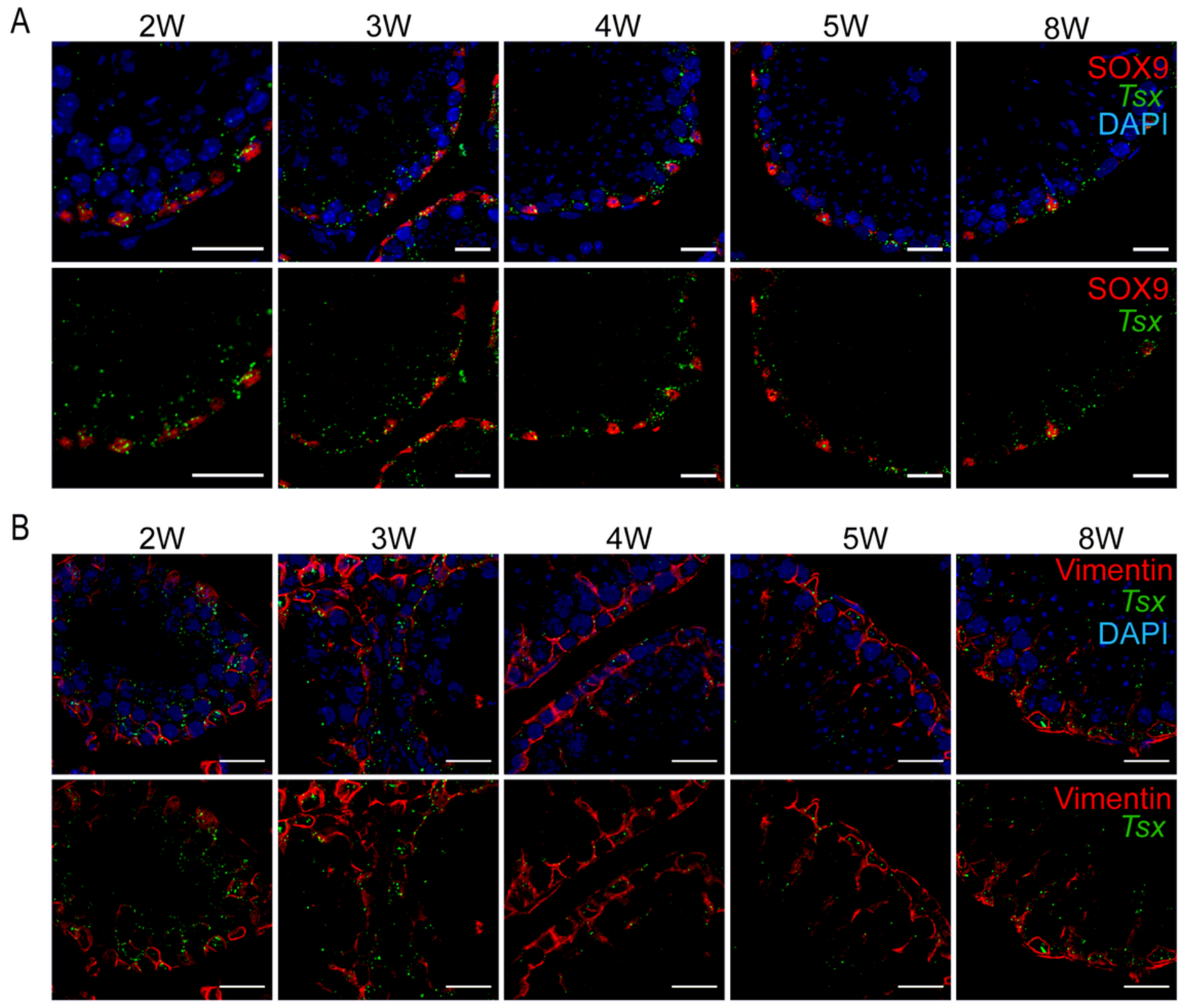

Figure 2

Localization pattern of Tsx in testis. (A) Tsx RNA (green) co-staining with nuclei marker of Sertoli cells SOX9 (red). Testes collected from 2 to 8 week-old mice were fixed in 4\% PFA, paraffin embedded and then sectioned. Nuclei were stain with DAPI. Scale bar, $20 \mu \mathrm{m}$. (B) Tsx RNA (green) co-staining with cytoplasm marker of Sertoli cells Vimentin (red). Nuclei were stained with DAPI. Testes were treated the same as that in (A). Scale bar, $20 \mu \mathrm{m}$. 
A

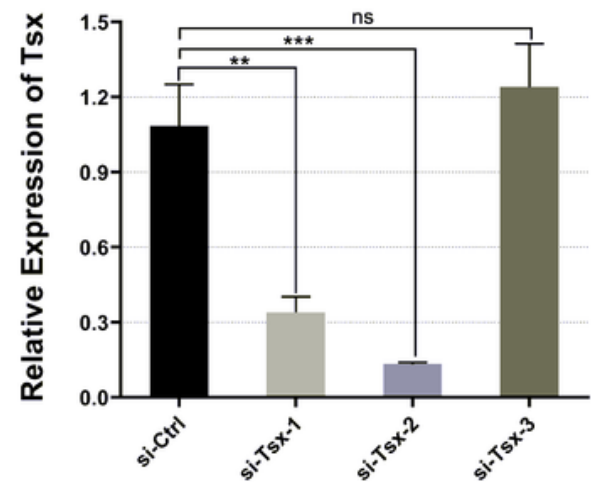

C

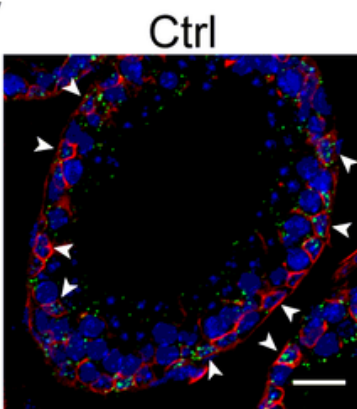

E
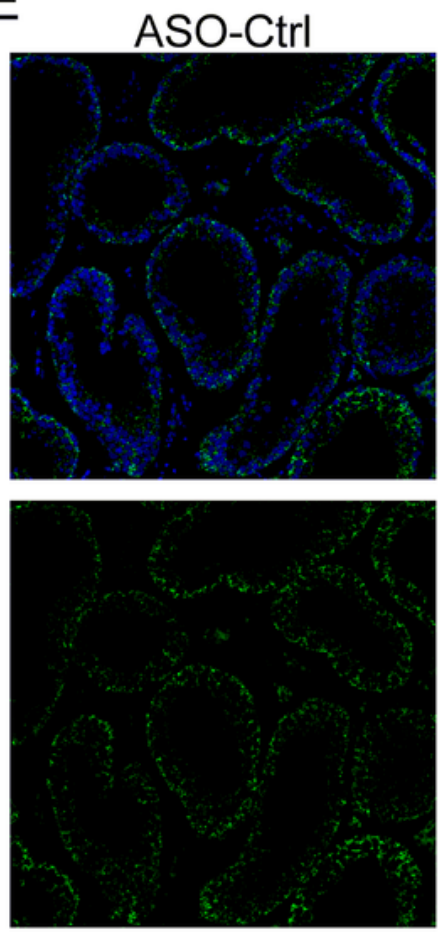

B

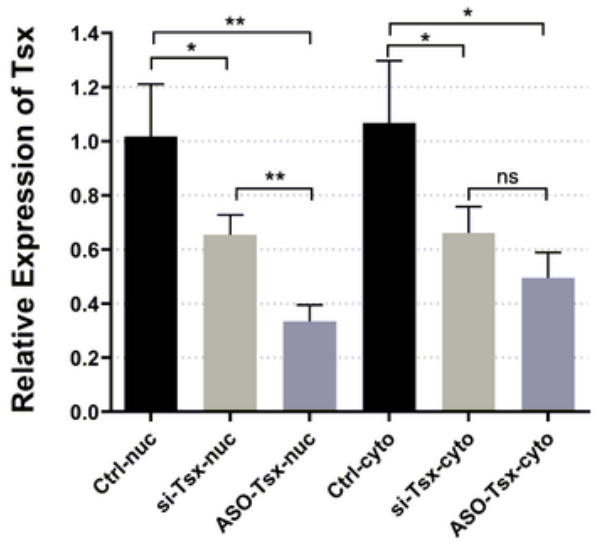

si-Tsx-2

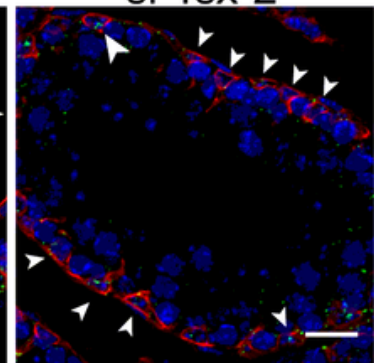

ASO-Tsx-2
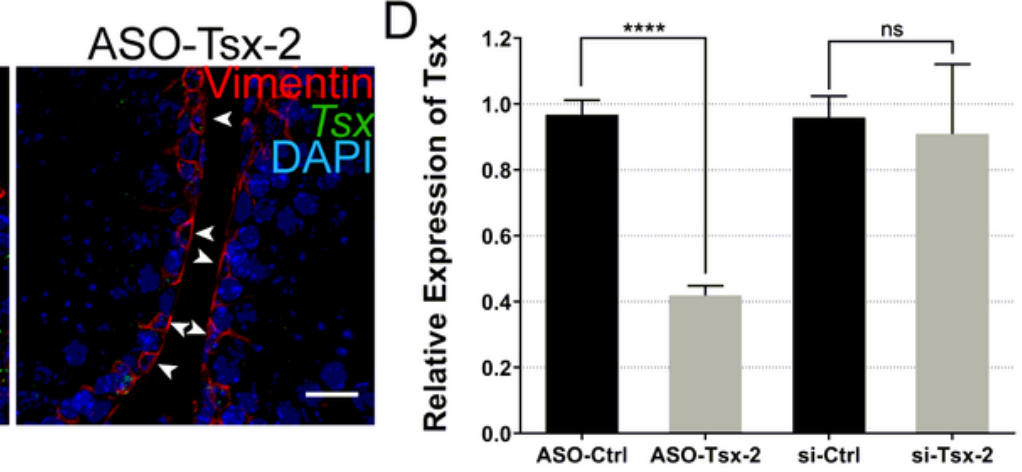

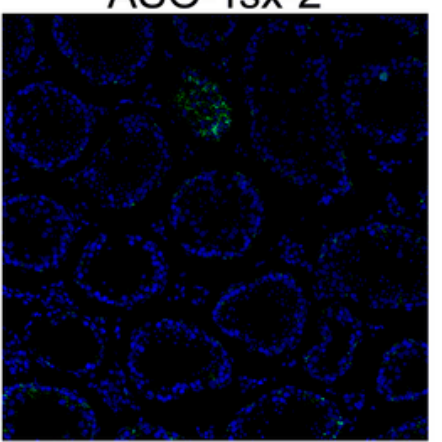

si-Ctrl

si-Tsx-2

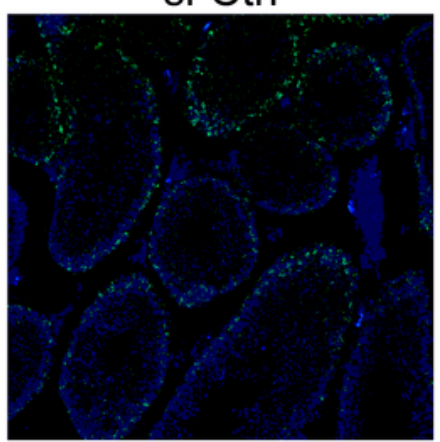

TSX

DAPI
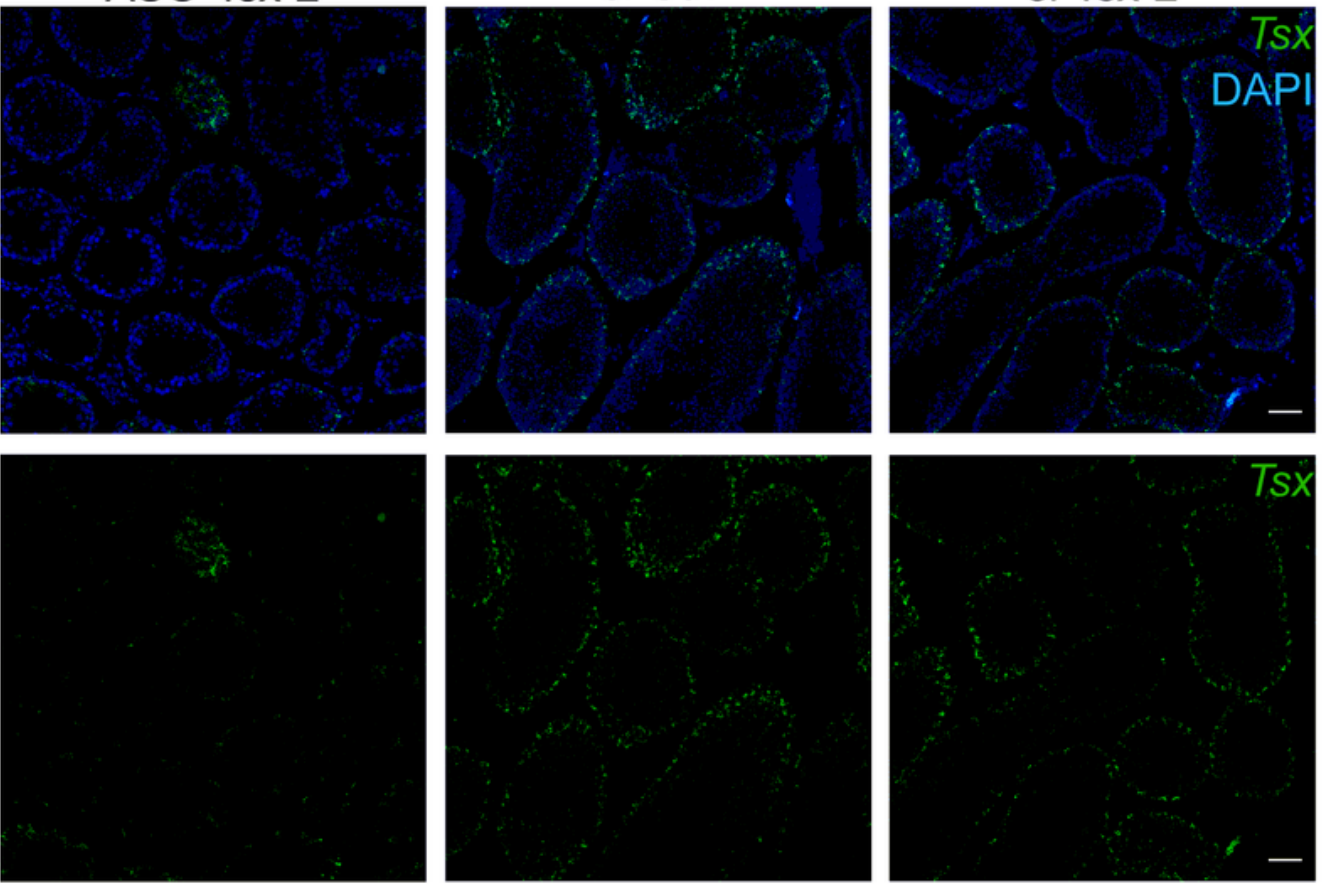

TsX

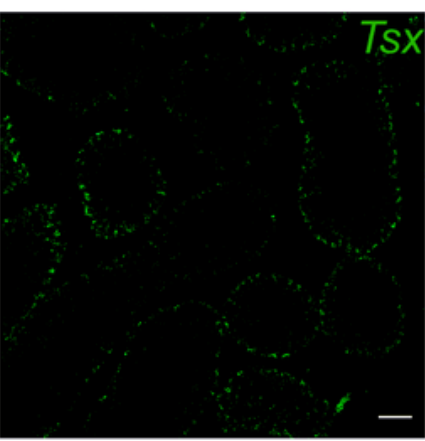

Figure 3

Comparative evaluations of ASOs and siRNAs in testis. (A) The RNA level of Tsx detected by RT-PCR in control and knockdown testis treated with three different siRNAs. ${ }^{* \star} p<0.01,{ }^{*} * x p 0.001, n=3$. (B) RT-PCR analysis of Tsx expression in cytoplasm and nucleus 2 days after injection of si-Tsx-2 or ASO-Tsx-2. ${ }^{*} p<0.05,{ }^{*} p<0.01, n=3$. (C) FISH of Tsx RNA (green) with IF of Vimentin (red). Testes were collected 2 days after injection of siRNA or ASO. Nuclei were stained with DAPI. Nuclei of Sertoli cells were indicated 
by white arrows. Scale bar, $20 \mu \mathrm{m}$. (D) RT-PCR results of Tsx RNA level 10 days after injection of si-Tsx-2 or ASO-Tsx-2. ${ }^{* * *} p<0.0001, n=3$. (E) FISH images of Tsx RNA in testes 10 days after injection of si-Tsx-2 or ASO-Tsx-2. Nuclei were stained with DAPI. Scale bar, $50 \mu \mathrm{m}$. (A and B) (D and E) Values are expressed as mean $\pm S D$. Statistical significance was determined using t tests.
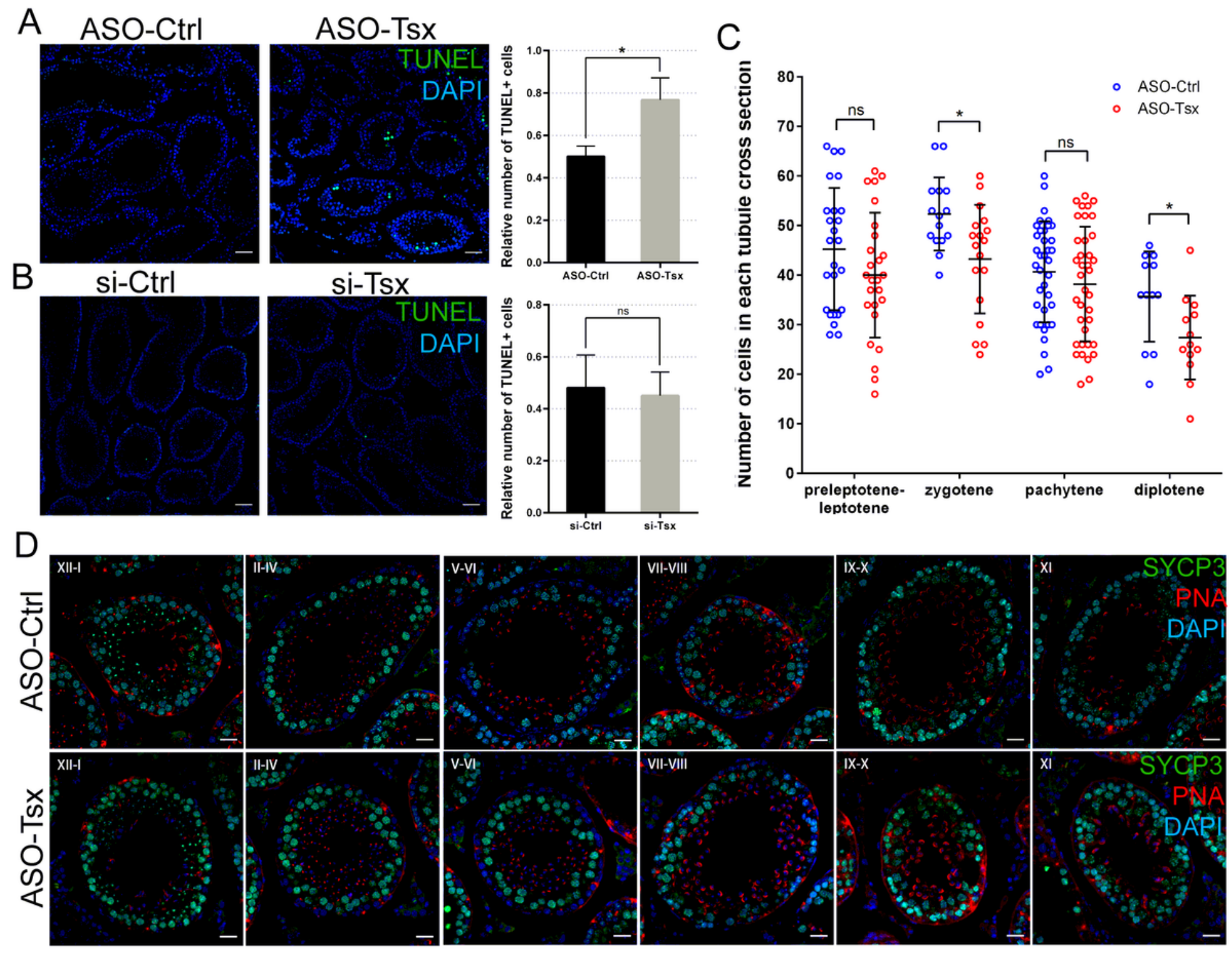

\section{Figure 4}

Physiological outcomes of Tsx ASOs in testis. (A and B) TUNEL staining and quantification of TUNELpositive cells in tubules from Tsx-knockdown mice injected with ASOs (A) or siRNAs. (B) Nuclei were stained with DAPI. Scale bar, $50 \mu \mathrm{m}$. Relative number of TUNEL+ cells is determined by counting the number of TUNEL-positive cells per field and dividing by the number of tubules in each field. A total of 150 tubules from ASO-mediated Tsx-knockdown testes and 100 tubules from siRNA-mediated Tsxknockdown testes were counted. Each contains three independent samples. (C) Quantification of four cell populations between control and Tsx-knockdown testes. Number of each cell population per tubule is shown in dot. 81 tubules from 3 testes injected with ASO-Tsx and 83 tubules from 3 testes injected with control ASO were analyzed. (D) Immunostaining of SYCP3 (green) and PNA (red) on testis sections from 
control and Tsx-knockdown mice. Seminiferous tubule sections were staged by morphology of chromosome and acrosome. Scale bar, $20 \mu \mathrm{m}$. (A-C) Values are expressed as mean \pm S.D. Statistical significance was determined using $t$ tests.

\section{Supplementary Files}

This is a list of supplementary files associated with this preprint. Click to download.

- Additionalfile1.pdf

- Additionalfile2.pdf 